\title{
Unusual Oxidation Behaviour of Mesoporous Silicates towards Lignin Model Phenolic Monomer
}

\author{
Rojalin Sadual1', Sushanta K. Badamali1 ${ }^{*}$, Sudhir E. Dapurkar², Rajesh K. Singh ${ }^{3}$ \\ ${ }^{1}$ Department of Chemistry, Utkal University, Bhubaneswar, India \\ ${ }^{2}$ Tata Chemicals Limited, Pune, India \\ ${ }^{3}$ Department of Chemistry, North Orissa University, Baripada, India \\ Email: ${ }^{*}$ skbadamali@gmail.com
}

Received 7 July 2015; accepted 5 September 2015; published 8 September 2015

Copyright (C) 2015 by authors and Scientific Research Publishing Inc.

This work is licensed under the Creative Commons Attribution International License (CC BY).

http://creativecommons.org/licenses/by/4.0/

c) (i) Open Access

\begin{abstract}
Oxidation of the lignin model monomer apocynol, 1-(4-hydroxy-3-methoxyphenoxy)-ethanol catalysed by mesoporous silica catalysts i.e. MCM-41, MCM-48, SBA-15 using $\mathrm{H}_{2} \mathrm{O}_{2}$ as an oxidant has been studied. Selectively, 2-methoxybenzoquinone was obtained along with acetovanillone. Such unprecedented oxidation behaviour of these metal free siliceous catalysts is attributed to the polar internal surface, high surface area as well as the pore architecture. On the other hand, the studied reaction was found to be non-selective when a commercial grade mesoporous silica i.e. Silica-5 was used as catalyst for comparison. Among the various silica catalysts studied, MCMs gave highest conversion and selectivity towards 2 -methoxybenzoquinone under very mild reaction conditions.
\end{abstract}

\section{Keywords}

Lignin, Apocynol, Methoxybenzoquinone, Acetovanillone, Mesoporous Silica

\section{Introduction}

The discovery of ordered mesoporous silicate materials by the Mobil researchers in 1992 [1] with high surface area $\left(700-1500 \mathrm{~m}^{2} \cdot \mathrm{g}^{-1}\right)$, tuneable pore size $(2-20 \mathrm{~nm})$, and large pore volume $\left(0.4-1.0 \mathrm{~cm}^{3} \cdot \mathrm{g}^{-1}\right)$ has attracted great interest for their potential applications in the fields of catalysis, chromatography, sensing, shape selective catalysis and support material [2] [3]. Since their discovery, two of the stable members of this family, MCM-41

${ }^{*}$ Corresponding author.

How to cite this paper: Sadual, R., Badamali, S.K., Dapurkar, S.E. and Singh, R.K. (2015) Unusual Oxidation Behaviour of Mesoporous Silicates towards Lignin Model Phenolic Monomer. World Journal of Nano Science and Engineering, 5, 88-95. 
having unidimensional pore structure and MCM-48 with three-dimensional pore system have been exploited extensively in the field of catalysis. The scope of application of mesoporous materials is further broadened with the advent of SBA-n molecular sieve and SBA-15 in particular is being widely studied [4]. However, studies on the use of metal free siliceous materials in catalysis are rare and limited. Particularly, purely siliceous framework possesses substantial amounts ( 30\% - 40\%) of silanol (defect sites) groups [5]. And various active functionalities have often been implanted via these hydroxyl groups to enable these silicates to be catalytically active. Notably few interesting reports are available on the oxidation behaviour of silica based materials. Morasas and Harrington have reported the oxidative and hydroxylative conversions of polar aromatic compounds over quartz surface [6]. And the peroxidative activity of silica is chiefly attributed to the polar nature of the silica surface [7]. Similarly, the photocatalytic decarboxylation of organic compounds catalyzed in the presence of mesoporous silicas has studied by Itoh et al. [8]. Recently, we have reported the oxidative ability of mesoporous silica materials towards lignin model compound under microwave irradiation [9] [10]. It has been demonstrated that $\alpha$ methylvanillyl alcohol (apocynol) can selectively be transformed to either acetovanillone or 2-methoxybenzoquinone, using mesoporous silica and $\mathrm{H}_{2} \mathrm{O}_{2}$ as oxidant under microwave activation. These motivating results further stimulated us to continue the study on the reaction under conventional heating rather than microwave activation, as the former was less expensive and industrially important.

Among the lignin model phenolic monomer, Apocynol, 1-(4-hydroxy-3-methoxyphenoxy)-ethanol is commonly studied over different catalysts in order to understand the reaction pathway, which can possibly be extended to lignin based biomass. Recently, much attention is focussed on selective transformation of lignin derived fragments to fine chemicals through catalysis [11]. Wozniak et al. [12] have studied the liquid phase oxidation of apocynol over potassium nitrosodisulfonate (Fremy's salt) and corresponding benzoquinones were selectively ( $\sim 0 \%$ ) obtained over a reaction period of $2 \mathrm{~h}$. Similarly, 2-methoxybenzoquinone was obtained as the common product with maximum selectivity of $50 \%$ by using Co(salen) catalysts [13] [14]. Realising the importance of benzoquinones as precursors in various organic reactions, currently the possible generation of quinones from lignin based guaiacyl units is being investigated [15]. In the present study, we report on the unusual oxidation behaviour of the siliceous mesoporous materials towards apocynol using $\mathrm{H}_{2} \mathrm{O}_{2}$ as an oxidant under mild reaction conditions with highest selectivity towards 2-methoxybenzoquinone. For a comparison commercial grade mesoporous silica (Silica-5, Grace, Germany) was also used as catalyst as received.

\section{Experimental}

\subsection{Preparation of Mesoporous Molecular Sieve}

Mesoporous silica catalysts; MCM-48, MCM-41 and SBA-15 were synthesized hydrothermally as per the procedure previously reported [1] [4] [16].

For the synthesis of MCM-48, $0.67 \mathrm{~g}$ of sodium hydroxide was dissolved in $17 \mathrm{ml}$ of distilled water and stirred. In another beaker, $7.11 \mathrm{~g}$ of hexadecyltrimethylammonium bromide was dissolved in $28.4 \mathrm{ml}$ of distilled water and stirred for $30 \mathrm{~min}$. Then, $7.1 \mathrm{ml}$ of tetraethyl orthosilicate was added to the sodium hydroxide solution with continuous stirring. To this mixture, the surfactant solution was added slowly under stirring conditions and was left out for 30 min so that a uniform solution is formed. The gel was then transferred to a teflon bottle and aged at $373 \mathrm{~K}$ for $72 \mathrm{~h}$. The solid product obtained was recovered by repeated washing followed by drying in air oven at $352 \mathrm{~K}$ for $6 \mathrm{~h}$. The template was removed by calcining at $823 \mathrm{~K}$ for $3 \mathrm{~h}$ in flow of nitrogen followed by $3 \mathrm{~h}$ in air.

Mesoporous silica SBA-15 was synthesized in an acidic medium with poly(alkylene oxide) triblock copolymers, such as poly(ethylene oxide)-poly(propylene oxide)-poly(ethylene oxide) (PEO-PPO-PEO) as the surfactant, tetraethyl orthosilicate as a source of silica. A total of $4.0 \mathrm{~g}$ of Pluronic123 was first added to $120 \mathrm{~mL}$ of 2 $\mathrm{M} \mathrm{HCl}$ along with $30 \mathrm{~mL}$ of deionised water under constant stirring until a clear solution was obtained. To this $8.5 \mathrm{~g}$ of tetraethyl orthosilicate was added followed by continuous stirring at $313 \mathrm{~K}$ for $24 \mathrm{~h}$. The gel was transferred to a teflon bottle and aged at $373 \mathrm{~K}$ for $24 \mathrm{~h}$. The solid product was recovered by filtration and repeated washing with deionised water, followed by drying at $343 \mathrm{~K}$ for overnight. The template was removed by calcining at $823 \mathrm{~K}$ for $6 \mathrm{~h}$ in air.

The synthesis of MCM-41 was carried out, in a basic medium with hexadecyltrimethylammonium bromide (CTAB) as surfactant and sodium silicate as the silica source. In a typical synthesis procedure, $40 \mathrm{~g}$ of water, $18.7 \mathrm{~g}$ of sodium silicate, $1.2 \mathrm{~g}$ of sulfuric acid were combined under continuous stirring. The resulting mixture 
was then allowed for stirring for $15 \mathrm{~min}$. To this, the surfactant solution, prepared by mixing 17.0 g CTAB with $50 \mathrm{ml}$ water, was added and stirred for $30 \mathrm{~min}$. Another $20 \mathrm{~g}$ of water was then added to this gel, after this, the resulting gel was crystallized at $373 \mathrm{~K}$ for $6 \mathrm{~d}$. The solid was then recovered by washing repeatedly followed by filtration. The recovered products were then dried at $353 \mathrm{~K}$ for $12 \mathrm{~h}$. The as-synthesized product was then calcined at $823 \mathrm{~K}$ for $3 \mathrm{~h}$ in flowing nitrogen, followed by $3 \mathrm{~h}$ in flowing air.

\subsection{Preparation of Apocynol}

Apocynol was synthesized as per the procedure outlined elsewhere [10] with some minor modifications and characterized by melting point, ${ }^{1} \mathrm{H},{ }^{13} \mathrm{C}$ NMR (Varian), GC (Varian) and GC-MS (Agilent) studies.

\subsection{Characterization of the Catalysts}

The synthesized materials were characterized by powder X-ray diffraction (XRD) and nitrogen physisorption measurements. XRD patterns were recorded using Brucker D8 diffractometer with Cu K $\alpha$ radiation. BET-surface area and pore size were measured by using ASAP 2020, Micromeritics analytical system.

\subsection{Catalytic Activity Studies}

In a typical catalytic reaction, apocynol (1.0 mmol, $168 \mathrm{mg})$, acetonitrile (5 mL), the catalyst (100 mg) and 35\% aqueous $\mathrm{H}_{2} \mathrm{O}_{2}(3.0 \mathrm{mmol}, 0.34 \mathrm{~mL})$ were placed in a round bottomed flask and allowed to react with varying reaction temperature and duration. The reaction mixture was analysed by GC (Varian-450 instrument), with VF-1ms column $(15 \mathrm{~m} \times 0.25 \mathrm{~mm} \times 0.39 \mathrm{~mm}$ film thickness) and flame ionization detector (FID). Substrate conversion and product selectivity were determined using an external standard method, in a similar procedure described earlier [10].

\section{Results and Discussion}

The XRD patterns of the calcined mesoporous silica catalysts are shown in Figure 1. XRD patterns of calcined, MCM-41 (Figure 1(a)) and MCM-48 (Figure 1(b)) show a strong reflection observed in between $2 \theta=2.2^{\circ}-3^{\circ}$ in addition to several weak reflections in the range of $2 \theta=3^{\circ}-7^{\circ}$ indicated the formation of mesoporous structure. Similarly, in case of SBA-15 (Figure 1(c)), a strong reflection at $2 \theta=1.06^{\circ}$ and several weak reflections between $2 \theta=1.5^{\circ}-3.0^{\circ}$ confirms its structure. The BET surface area and pore size were determined to be 978 $\mathrm{m}^{2} \cdot \mathrm{g}^{-1}$ and $2.6 \mathrm{~nm}$ for MCM-48 while that for MCM-41 and SBA-15 was found to be $850 \mathrm{~m}^{2} \cdot \mathrm{g}^{-1}, 2.5 \mathrm{~nm}$ and $940 \mathrm{~m}^{2} \cdot \mathrm{g}^{-1}, 6.1 \mathrm{~nm}$ respectively [17].

Figure 2 shows the FT-IR spectra of calcined MCM-41 (a) MCM-48 (b) and SBA-15 (c) respectively. All the calcined spectra, show absence of the characteristic band for surfactant template molecule at 2928, 2854 and $1490 \mathrm{~cm}^{-1}$ leaving behind other characteristic peaks of mesoporous material. In addition to above features, absorption bands noticed at around 1072, 808 and $460 \mathrm{~cm}^{-1}$ are originating from the asymmetric, symmetric stretching and bending vibration for Si-O-Si bonds [18] respectively. A strong band at $966 \mathrm{~cm}^{-1}$ seen clearly, in all the spectra, is attributed to terminal Si-O stretching of silanol groups. The occurrence of broad bands between $3000-3800 \mathrm{~cm}^{-1}$ in case of all the samples indicates the presence of surface hydroxyl groups, characteristic of mesoporous structure [19]. It is worth mentioning that both MCM-41 and MCM-48 show intense band in this range compared to SBA-15. This is attributed to the abundantly presence of incompletely condensed silica as evidenced from ${ }^{29} \mathrm{Si}$ MAS-NMR data [20]. The predominant presences of silanol (三SiOH) groups impart hydrophilic nature to these MCM-n type materials.

\section{Catalytic Experiments}

We have undertaken a detail investigation on the oxidation of apocynol, 1 (Scheme 1), catalyzed by different catalysts under varying reaction conditions such as temperature, substrate: oxidant, duration and the results are summarized in Table 1.

Over all the silica catalysts used, it was noticed that within a very short period (15 min) major substrate was reacted yielding both 2 and $\mathbf{3}$. Acetovanillone is the early oxidation product [21], and 2-methoxybenzoquinone is believed to be formed by the generation of phenoxy radical and subsequent cleavage of side chain [22]. 


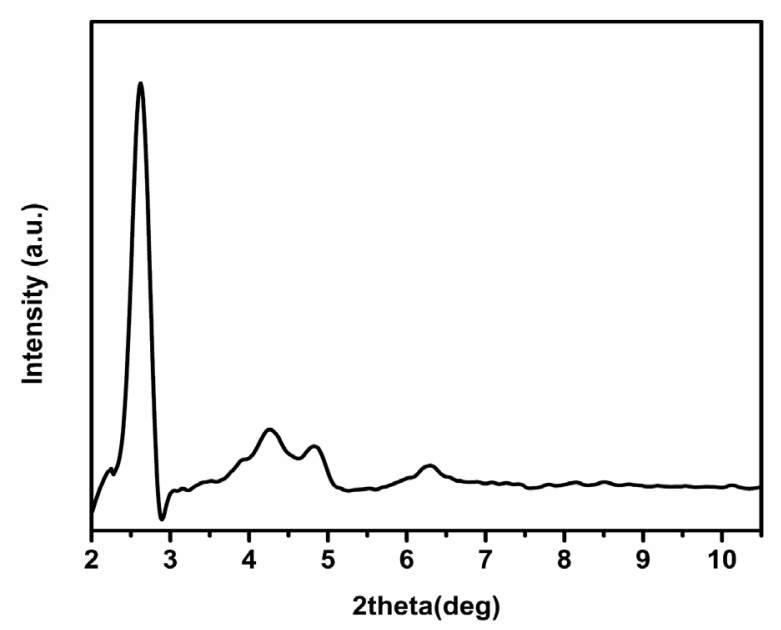

(a)

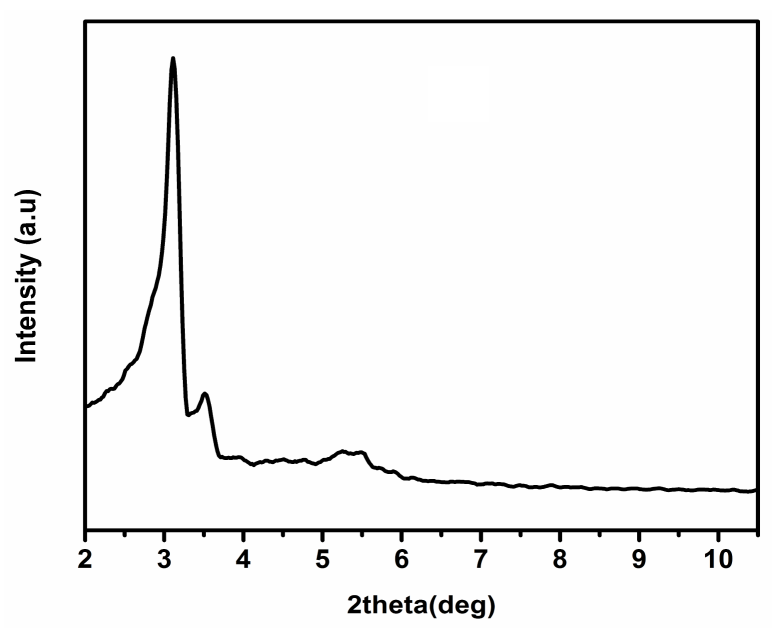

(b)

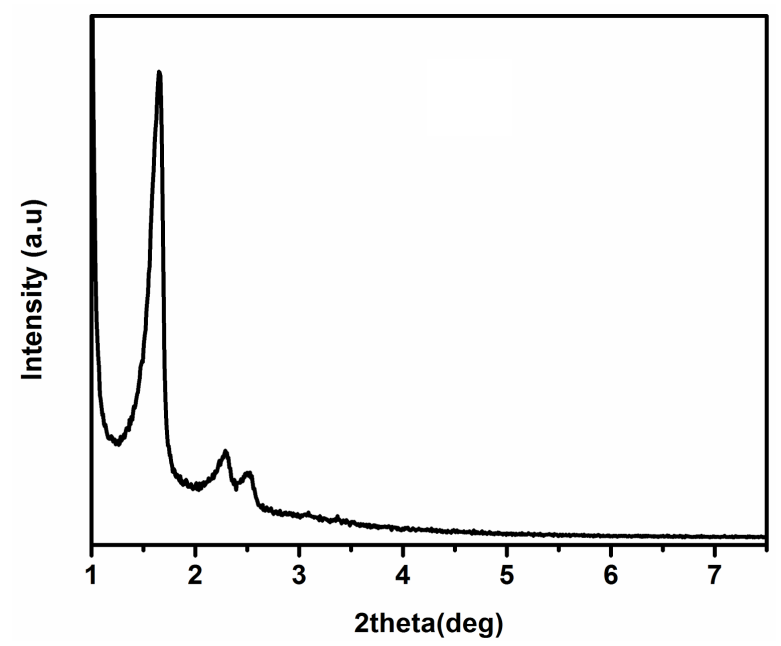

(a)

Figure 1. XRD patterns of calcined (a) MCM-41; (b) MCM-48 and (c) SBA-15.

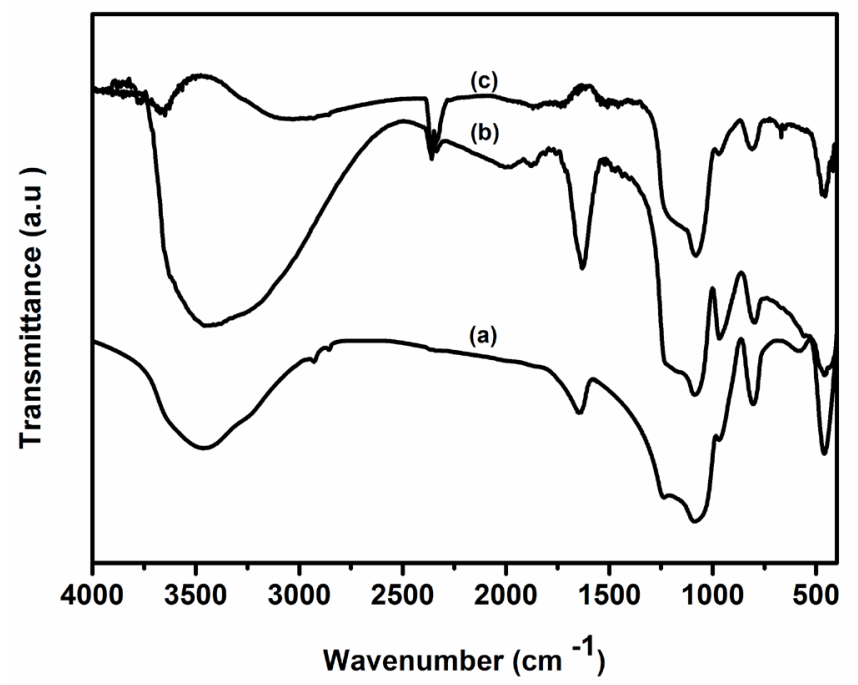

Figure 2. FT-IR spectra of calcined (a) MCM-41; (b) MCM-48 and (c) SBA-15. 


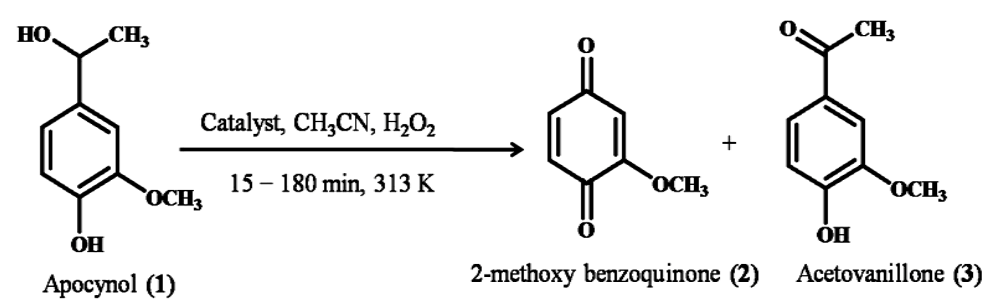

Scheme 1. Reaction profile of oxidation of apocynol (1).

Table 1. Reaction profile of oxidation of apocynol (1).

\begin{tabular}{ccccc}
\hline Entry & Catalyst & $\begin{array}{c}\text { Duration } \\
\text { (min) }\end{array}$ & & Product distribution \\
(\%)
\end{tabular}

Reaction conditions: $1.0 \mathrm{mmol}$ apocynol, $3 \mathrm{mmol} \mathrm{H} \mathrm{O}_{2}, 5 \mathrm{~mL}$ acetonitrile, $100 \mathrm{mg}$ catalyst at $313 \mathrm{~K}$, Duration 15 - $180 \mathrm{~min}$.

Interestingly, about $98 \%$ of substrate conversion was achieved over MCM-41 with best quinone selectivity ( $\sim 84 \%)$ among the catalysts screened. In general an optimum conversion and selectivity was obtained within 30 min of reaction. Continuing the reactions beyond 30 min resulted in an increase of substrate conversion with fall in selectivity, when MCM-48 and SBA-15 were used as catalysts. However, MCM-41 maintained a steady increase in conversion and selectivity.

It is well evident that apocynol is quite stable irrespective of $3 \mathrm{~h}$ of heating in the solvent (entry-6). And in presence of $\mathrm{H}_{2} \mathrm{O}_{2}$ (entry-5), 1 showed high conversion resulting in 2-methoxybenzoquinone (2), acetovanillone (3) along with high molecular weight (unidentified) products. Additional products detected in the higher retention time, are attributed to the possible polymerisation of quinone to form high molecular weight compounds. In order to understand the influence of nature of silica and porous structure, Silica-5 having high surface area with irregular porous structure was employed as catalyst, which gave a lower substrate conversion with nearly equal selectivity towards 2-methoxybenzoquinone and acetovanillone (entry-4). These results indicated the fact that an ordered porous silicate surface as well as the pore architecture governs both the conversion and selectivity.

Another significant feature of this reaction has been, with increase in reaction duration the apocynol conversion remained almost same, while significant fall in acetovanillone yield. In order to understand this aspect, we further investigated the reaction profile by varying substrate: oxidant amount. Representative results of 
MCM-48 catalyst are presented in Table 2. It is well evident that with increase in the oxidant amount the selectivity of the 2-methoxybenzoquinone was enhanced with drop in acetovanillone yield. These observations point toward that 2-methoxybenzoquinone is the stable product which is formed at the expense of acetovanillone and similar trend was observed over other catalysts studied. Based on the results obtained, the product formation is likely to occur through a plausible pathway (Scheme 2). The polar surface along with high surface area favours the adsorption of both apocynol and hydrogen peroxide $\left(\mathrm{H}_{2} \mathrm{O}_{2}\right)$. Subsequently, binding of $\mathrm{H}_{2} \mathrm{O}_{2}$ onto the $-\mathrm{OH}$ group leads to activation of the oxidant resulting in possible formation of hydroxyl radicals. The hydroxyl radical formed either abstracts a phenolic hydrogen atom from the phenolic substrate, $\mathbf{1}$ (path-1) and produces a reactive phenoxy radical, $\mathbf{4}$ or it abstracts a proton from the benzylic carbon (path-2), forming the reactive radical, 6. By the path-1, 4, transformed to intermediate, 5, through addition of another hydroxyl radical. Intermediate $\mathbf{5}$, undergoes rearrangement to form 2-methoxybenzoquinone, 2 . On the other hand, through path-2, intermediate 6 undergoes deprotonation via another hydroxyl radical leading to the formation of acetovanillone, $\mathbf{3}$.

The unlikely oxidation behaviour of the mesoporous silicas is attributed to the abundance of surface hydroxyl groups, polar internal surface, high surface area and pore geometry. At this juncture the facts suggest that,
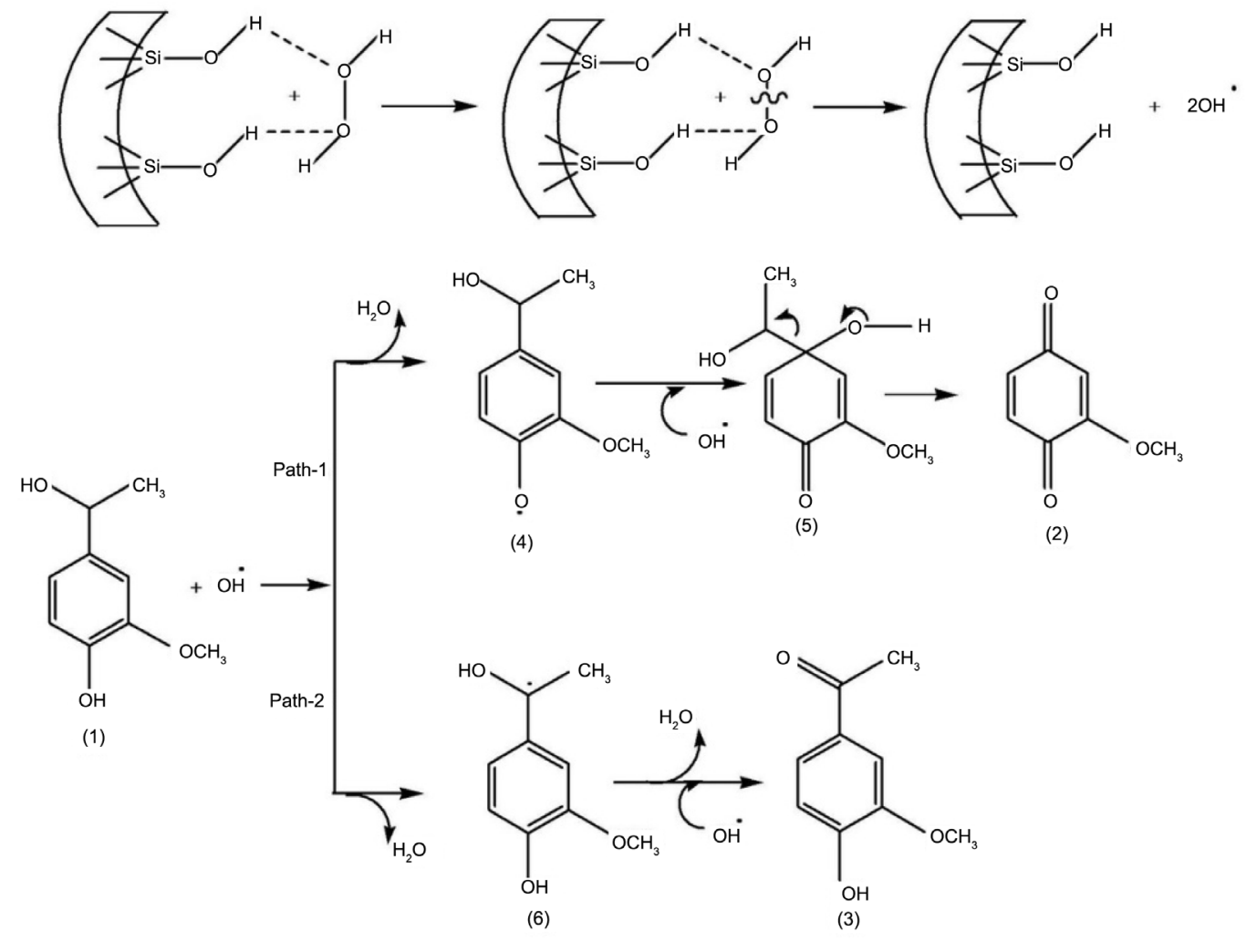

Scheme 2. Probable oxidation pathway of (1) over silica catalysts.

Table 2. Reaction profile of oxidation of apocynol (1) by varying oxidant amount over MCM-48.

\begin{tabular}{ccccc}
\hline Entry & Substrate:oxidant amount & $\begin{array}{c}\text { Substrate (1) } \\
\text { conv. (\%) }\end{array}$ & $\begin{array}{c}\text { Product (2) } \\
\text { yield (\%) }\end{array}$ & $\begin{array}{c}\text { Product (3) } \\
\text { yield (\%) }\end{array}$ \\
\hline 1 & In absence of $\mathrm{H}_{2} \mathrm{O}_{2}$ & 30 & - & - \\
2 & Sub: $\mathrm{H}_{2} \mathrm{O}_{2}=1: 1$ & 58 & 30 & 29 \\
3 & Sub: $\mathrm{H}_{2} \mathrm{O}_{2}=1: 2$ & 56 & 70 & 18 \\
4 & Sub: $\mathrm{H}_{2} \mathrm{O}_{2}=1: 3$ & 94 & 67 & - \\
\hline
\end{tabular}

Reaction conditions: $1.0 \mathrm{mmol}$ apocynol, $5 \mathrm{~mL}$ acetonitrile, $100 \mathrm{mg}$ catalyst at $313 \mathrm{~K}$, duration $180 \mathrm{~min}$. 
apocynol (1) is susceptible to oxidation in presence of $\mathrm{H}_{2} \mathrm{O}_{2}$ alone; and the reaction proceeds in an uncontrolled way resulting in several high molecular weight products (not identified). On the other hand, mesoporous silicates along with $\mathrm{H}_{2} \mathrm{O}_{2}$ catalytic system produced 2-methoxybenzoquinone predominantly, and the reaction was much controlled over silicate catalysts and hydrogen peroxide $\left(\mathrm{H}_{2} \mathrm{O}_{2}\right)$ oxidant system.

\section{Conclusion}

In conclusion, we have demonstrated a clean, efficient catalyst system for the selective oxidation of an important lignin model phenolic monomer. Metal free mesoporous silicas along with environmentally benign oxidant, $\mathrm{H}_{2} \mathrm{O}_{2}$, were found to be highly efficient catalytic system for the chosen oxidation reaction, and quinone was selectively obtained under mild reaction conditions. These results open up for further study to evaluate and understand the oxidative nature of mesoporous amorphous silica materials.

\section{Acknowledgements}

We wish to thank Department of Science and Technology, Govt. of India for the financial support under FAST track (SR/FT/CS-93/2007) and PURSE scheme. Authors appreciate many meaningful discussions with Dr. A. Sakthivel, Department of Chemistry, Delhi University, relating the reaction.

\section{References}

[1] Beck, J.S., Vartuli, J.C., Roth, W.J., Leonowicz, M.E., Kresge, C.T., Schmitt, K.D., Chu, C.T.W., Olson, D.H., Sheppard, E.W., McCullen, S.B., Higgins, J.B. and Schlenker, J.L. (1992) A New Family of Mesoporous Molecular Sieves Prepared with Liquid Crystal Templates. Journal of the American Chemical Society, 114, 10834-10843. http://dx.doi.org/10.1021/ja00053a020

[2] Fryxell, G.E. and Liu, J. (2000) Adsorption on Silica Surfaces. In: Papirer, E., Ed., Designing Surface Chemistry in Mesoporous Silica, Marcel Dekker, Inc., New York.

[3] Cao, G. (2004) Nanostructures and Nanomaterials. Synthesis, Properties, and Applications. Imperial College Press, London. http://dx.doi.org/10.1142/9781860945960

[4] Zhao, D., Huo, Q., Feng, J., Chmelka, B.F. and Stucky, G.D. (1998) Nonionic Triblock and Star Diblock Copolymer and Oligomeric Surfactant Syntheses of Highly Ordered, Hydrothermally Stable, Mesoporous Silica Structures. Journal of the American Chemical Society 120, 6024-6036. http://dx.doi.org/10.1021/ja974025i

[5] Selvam, P. and Dapurkar, S.E. (2004) Catalytic Activity of Highly Ordered Mesoporous VMCM-48. Applied Catalysis A: General, 276, 257-265. http://dx.doi.org/10.1016/j.apcata.2004.08.012

[6] Marasas, L.W. and Harrington, J.S. (1960) Some Oxidative and Hydroxylative Actions of Quartz: Their Possible Relationship to the Development of Silicosis. Nature, 188, 1173-1174. http://dx.doi.org/10.1038/1881173a0

[7] Schofield, P.J., Ralph, B.J. and Green, J.H. (1964) Mechanisms of Hydroxylation of Aromatics on Silica Surfaces. Journal of Physical Chemistry, 68, 472-476. http://dx.doi.org/10.1021/j100785a006

[8] Itoh, A., Kodama, T., Masuki, Y. and Inagaki, S. (2006) Oxidative Photo-Decarboxylation in the Presence of Mesoporous Silicas. Chemical and Pharmaceutical Bulletin, 54, 1571-1575. http://dx.doi.org/10.1248/cpb.54.1571

[9] Badamali, S.K., Luque, R., Clark, J.H. and Breeden, S.W. (2013) Unprecedented Oxidative Properties of Mesoporous Silica Materials: Towards Microwave-Assisted Oxidation of Lignin Model Compounds. Catalysis Communications, 31, 1-4. http://dx.doi.org/10.1016/j.catcom.2012.11.006

[10] Badamali, S.K., Clark, J.H. and Breeden, S.W. (2008) Microwave Assisted Selective Oxidation of Lignin Model Phenolic Monomer over SBA-15. Catalysis Communications, 9, 2168-2170. http://dx.doi.org/10.1016/j.catcom.2008.04.012

[11] Holladay, J.E., Bozell, J.J., White, J.F. and Johnson, D. (2007) Top Value-Added Chemicals from Biomass, Results of Screening for Potential Candidates from Biorefinery Lignin, II, USDOE, PNNL-16983.

[12] Wozniak, J.C., Dimmel, D.R. and Malcom, E.W. (1990) The Generation of Quinones from Lignin and Lignin-Related Compounds, Diels-Adler Reactions of Lignin-Derived Quinones, Lignin-Derived Quinones as Pulping Additives. Institute of Paper Science and Technology Paper Series No. 349, 1-57.

[13] Cedeno, D. and Bozell, J.J. (2012) Catalytic Oxidation of Para-Substituted Phenols with Cobalt-Schiff Base Complexes $/ \mathrm{O}_{2}$ - Selective Conversion of Syringyl and Guaiacyl Lignin Models to Benzoquinones. Tetrahedron Letters, 53, 2380-2383. http://dx.doi.org/10.1016/j.tetlet.2012.02.093

[14] Canevali, C., Orlandi, M., Pardi, L., Rindone, B., Scotti, R., Sipila, J. and Morazzone, F. (2002) Oxidative Degradation 
of Monomeric and Dimeric Phenylpropanoids: Reactivity and Mechanistic Investigation. Journal of the Chemical Society, Dalton Transactions, No. 15, 3007-3014. http://dx.doi.org/10.1039/b203386k

[15] Biannic, B. and Bozell, J.J. (2013) Efficient Cobalt-Catalyzed Oxidative Conversion of Lignin Models to Benzoquinones. Organic Letters, 15, 2730-2733. http://dx.doi.org/10.1021/ol401065r

[16] Schmidt, R., Stocker, M., Akporiaye, D., Torstad, E.H. and Olsen, A. (1995) High-Resolution Electron Microscopy and X-Ray Diffraction Studies of MCM-48. Microporous Materials, 5, 1-7. http://dx.doi.org/10.1016/0927-6513(95)00030-D

[17] Sadual, R. (2015) Oxidation of Lignin Model Phenolic Monomer over Cobalt Containing Solid Catalysts. PhD Thesis, North Orissa University, Baripada.

[18] Flanigen, E.M., Khatami, H. and Szymanski, H.A. (1971) Infrared Structural Studies of Zeolite Frameworks. In: Flanigen, E.M. and Sand, L.B., Ed., Molecular Sieve Zeolites, Advances in Chemistry 101, American Chemical Society, Washington DC, 201-229. http://dx.doi.org/10.1021/ba-1971-0102

[19] Chen, J.S., Li, Q.H., Xu, R.R. and Xiao, F.S. (1995) Distinguishing the Silanol Groups in the Mesoporous Molecular Sieve MCM-41. Angewandte Chemie International Edition in English, 34, 2694-2696. http://dx.doi.org/10.1002/anie.199526941

[20] Zhao, X.S., Lu, G.Q., Whittaker, A.K., Millar, G.J. and Zhu, H.Y. (1997) Comprehensive Study of Surface Chemistry of MCM-41 Using ${ }^{29}$ Si CP/MAS NMR, FTIR, Pyridine-TPD, and TGA. The Journal of Physical Chemistry B, 101, 6525-6531. http://dx.doi.org/10.1021/jp971366+

[21] Crestini, C., Pastoni, A. and Taguatesta, P. (2004) Metalloporphyrins Immobilized on Motmorillonite as Biomimetic Catalysts in The Oxidation of Lignin Model Compounds. Journal of Molecular Catalysis A: Chemical, 208, 195-202. http://dx.doi.org/10.1016/j.molcata.2003.07.015

[22] Sadual, R., Badamali, S.K. and Singh, R.K. (2014) Studies on Mesoporous CoSBA-15 Catalysed Selective Oxidation of a Lignin Model Phenolic Monomer. Advanced Porous Materials, 2, 48-53. http://dx.doi.org/10.1166/apm.2014.1044 